\title{
Théologiques
}

\section{Note sur pouvoir et interprétation}

\section{Walter Moser}

Volume 8, numéro 2, automne 2000

Le pouvoir

URI : https://id.erudit.org/iderudit/005018ar

DOI : https://doi.org/10.7202/005018ar

Aller au sommaire du numéro

Éditeur(s)

Faculté de théologie de l'Université de Montréal

ISSN

1188-7109 (imprimé)

1492-1413 (numérique)

Découvrir la revue

Citer ce document

Moser, W. (2000). Note sur pouvoir et interprétation. Théologiques, 8(2), 99-106. https://doi.org/10.7202/005018ar d'utilisation que vous pouvez consulter en ligne.

https://apropos.erudit.org/fr/usagers/politique-dutilisation/ 
Théologiques 8/2 (2000) 99-106

\section{Note sur pouvoir et interprétation}

Walter MOSER

Département de littérature comparée Université de Montréal

L'enjeu du pouvoir est indissociable de l'acte d'interprétation, mais l'herméneutique ne l'a pas toujours explicité. Si on admet avec Gadamer que toute interprétation a lieu dans une situation concrète, qu'elle est même, sur un plan tout à fait pratique, une application concrète d'un texte à une situation, on peut facilement comprendre que le facteur pouvoir et exercice de pouvoir ne soit pas étranger au travail de l'interprète. Ce travail pose toujours - explicitement ou implicitement - un horizon de sens qui découle de la situation de l'interprétation. L'horizon de sens détermine la manière dont le potentiel de sens d'un texte sera actualisé dans un acte d'interprétation concret ; il opère l'ouverture d'un champ que pourra occuper, traverser, arpenter l'interprète, mais il trace aussi une limite à ne pas franchir : " au-delà de cet horizon, quelque vaste qu'il soit, pas de sens qui vaille, pas de salut herméneutique».

Une part importante du pouvoir impliqué dans l'acte interprétatif réside dans l'établissement de cet horizon de sens. Un pouvoir souvent caché, non assumé, parce qu'il s'inscrit dans les préalables de l'acte proprement dit.

Tel n'est cependant pas le cas lorsqu'il s'agit d'une interprétation explicitement dogmatique. Dans Le Magistère chrétien qui comporte la théorie de l'exégèse de la Bible par saint Augustin, le dogme établissant l'horizon à l'intérieur duquel la Bible peut prendre sens, est donné. L'ouvrage de saint Augustin est organisé en quatre livres, dont le premier s'intitule "Des vérités à découvrir dans l'Écriture ». C'est ici que le maître d'exégèse établit les principes de la foi chrétienne (vérité de la foi, vertu théologale de la charité, etc.) qui guideront plus 
tard l'interprète quand il aborde sa tâche de " dissiper les ambiguïtés de l'écriture » (cf. le titre du 3e livre). C'est à ces principes qu'il renvoie dans le troisième livre, qui contient l'herméneutique proprement dite, quand il s'agit de prendre des décisions interprétatives concrètes sur tel mot, sur tel passage, sur telle tournure du texte biblique.

L'exégète "doit consulter la règle de la foi qu'il a reçue de passages plus clairs de l'Écriture ou de l'autorité de l'Église, et dont nous avons traité au premier livre » (p 341). L'horizon de sens est donc bien tracé. Il guide l'exégète en circonscrivant la zone du sens; il admet même la chose que Roland Barthes a appelé le sens pluriel. Pour saint Augustin, en fait, un passage biblique peut avoir plusieurs sens, à condition toutefois qu'ils soient tous " non incompatibles avec la vérité de la foi »(p 389). Mais l'horizon de sens dogmatique devient aussi un garde-fou, au sens propre du terme, car de l'autre côté de la ligne tracée guette le non-sens, voire la démence : "Or, croire cela [une vérité non-conforme avec le dogme], serait le comble de la démence » (p 345). Cette ligne qui trace le champ de liberté de l'interprète est donc aussi celle qui exclut, d'ailleurs exactement selon un des principes d'exclusion retenus par Michel Foucault dans L'ordre $d u$ discours : la ligne tracée entre raison et folie, dont Foucault a bien montré les enjeux de pouvoir.

L'exercice de pouvoir qui habite ce tracé de l'horizon du sens devient encore plus évident si on observe la circularité de l'opération qui s'ensuit : au départ, en tant que texte sacré et premier du christianisme, la Bible est le fondement des valeurs et vérités théologales et plus généralement de la "vérité de la foi ". Cette vérité est alors érigée en horizon de sens pour l'interprétation du texte biblique, plus exactement des passages difficiles, car les passages qui confirment la vérité de la foi sont présumés compris par saint Augustin et lui servent alors de passages parallèles, selon une logique de cohérence interne du texte, pour établir le «bon » sens des passages ambigus. Le résultat de ce travail d'exégèse ne pourra que confirmer l'horizon de sens préétabli, etc.

Cette circularité de l'interprétation dogmatique peut nous faire sourire aujourd'hui. Mais imaginons des situations où elle est prise en charge par une institution. Que ce soit l'école d'exégèse centralisée d'une Église ou le Comité central d'un parti unique - évidemment révolutionnaire - d'un État moderne ou encore une des multiples 
Écoles freudiennes qui veulent gérer le texte fondateur de la psychanalyse, ce sera chaque fois le même scénario : le dogme - explicite ou implicite - subit un durcissement institutionnel. Le sens est produit et administré par des professionnels qui ont le statut de fonctionnaires et sont investis de l'autorité d'un appareil institutionnel. L'interprétation - en tout cas celle des textes considérés premiers - est ainsi insérée dans une structure institutionnelle conçue pour exercer du pouvoir. Georges Gusdorf a montré les débuts de cette logique de la gestion et du contrôle du sens dans l'établissement de la Bibliothèque d'Alexandrie.

Rien de moins étonnant, en conséquence, de voir circuler une opinion publique au sujet de l'herméneutique, une espèce de vulgate sur l'interprétation, qui consiste à associer intrinsèquement interprétation et establishment. Et qui, de ce fait, situe le travail de l'interprète toujours du côté des puissants. En court-circuitant les étapes du raisonnement, ce qui est le propre des doxas, on attribue alors à l'herméneutique un statut idéologiquement et politiquement conservateur sinon réactionnaire : l'interprète logerait toujours à l'enseigne des puissants. À condition de contribuer à consolider leur pouvoir, il en profiterait.

Ceci n'est, cependant, que la moitié de l'histoire, car il s'agit d'une histoire qui se raconte sur le mode antithétique. Si on peut exercer du pouvoir par l'établissement et la gestion du sens, et que le sens devienne un enjeu de pouvoir, il est logique qu'il devienne aussi le lieu précis où on peut faire émerger un contre-pouvoir. Prenons à titre d'exemple ce " petit roman » du dîner de Turin que raconte Jean-Jacques Rousseau et que Jean Starobinski a si magistralement interprété. Le moment décisif de cet épisode des Confessions est un acte d'interprétation : le domestique-précepteur, admis à la table de ses patrons aristocrates, saisit l'occasion qui lui est offerte de leur donner une leçon d'interprétation. Pour un bref moment, sa performance interprétative le met au-dessus de tous, l'ordre social hiérarchique s'en trouve renversé. Il y a du révolutionnaire dans son geste ponctuel. Inutile de dire que cela ne durera pas, que l'ordre de type ancien régime sera rétabli.

N'importe la suite de l'histoire de Rousseau, Starobinski en profite pour faire une extrapolation surprenante sur l'acte d'interprétation. Il commence par constater : "C'est donc une nouvelle force qui 
se manifeste, prête à se transformer en pouvoir politique " pour enchaîner "Au dîner de Turin nous assistons déjà à une petite révolution" ( p 127) et, plus loin, en généralisant, il parle de "l'homme du dehors, mais qui surcompense le désavantage de sa situation périphérique en capturant par l'interprétation les secrets du dedans» . Cela s'applique à Freud tant qu'à Rousseau : "Il promeut de la sorte, à son tour, une révolution par l'interprétation » (p 156). Et voilà qu'interpréter prend une valeur intrinsèquement révolutionnaire. Avec une telle affirmation nous nous trouvons aux antipodes de l'institutionnalisation conservatrice de l'interprétation. Et elle n'est pas moins vraie! En fait, étant donné le lien constitutif entre pouvoir et interprétation, il est tentant de faire de l'activité interprétative le détonateur d'un changement de régime : une pratique de réforme, de révolte, de dissidence, de révolution.

D'ailleurs, les exemples historiques ne manquent pas. Qu'on pense, évidemment, à la Réforme historique. Un des enjeux centraux de ce mouvement mené par Luther portait sur la gestion du texte sacré des chrétiens : son interprétation et sa traduction. De même, les dissidences et le révisionnisme à l'intérieur du monde socialiste sont basées sur une relecture ${ }^{1}$ de l'œuvre fondatrice de Marx et Engels. La nouveauté « révolutionnaire » de la psychanalyse de Lacan, n'est-elle pas issue d'une relecture de Freud? En littérature, c'est la réinterprétation des grands classiques qui ponctue les changements de paradigme et par-là l'histoire littéraire.

Chaque fois qu'on attribue à un texte un statut fondateur, qu'on l'érige en texte premier, la gestion de son sens devient un enjeu de pouvoir très sensible. Les institutions s'en saisissent, mais aussi les dissidents, les révoltés et les révolutionnaires. Ce n'est donc pas l'acte interprétatif qui, en soi, serait révolutionnaire ou réactionnaire. Mais, comme il est indissociable de l'exercice du pouvoir, toutes sortes de pouvoirs s'en saisissent, s'en servent et s'y inscrivent pour s'affirmer. La question de savoir si le pouvoir de l'interprète se situe sur le versant conservateur ou innovateur, n'est pas intrinsèquement inscrite dans l'acte d'interprétation, mais dans la situation historique, sociale, poli-

1. Au sens fort dans lequel on a pris l'habitude de prendre ce terme: relire = réinterpréter, offrir une nouvelle interprétation, souvent contestatrice des dogmes interprétatifs établis. 
tique, économique concrète dans laquelle l'interprète intervient et à laquelle il applique son travail.

La question du révisionnisme est donc étroitement liée à celle de l'interprétation. Le terme a une connotation négative, puisqu'il a été créé par ceux qui détiennent un pouvoir institutionnel — dans la plupart des cas dans le contexte de l'état moderne - pour discréditer justement ceux qui s'attaquent à ce pouvoir en proposant une lecture alternative d'un texte fondateur. Dans le domaine littéraire, le critique et théoricien Harold Bloom a revalorisé ce terme en proposant le paradigme positif d'une critique révisionniste. Ce qui nous intéresse ici, c'est le moment révisionniste qu'il intègre dans sa théorie de la poésie. Selon Bloom, la production de la poésie a lieu dans le contexte œdipéen qui voit un poète-fils - faible par définition - devoir faire face à un poète-père puissant. Le nouveau poète subit donc l'anxiété de l'influence de la grande œuvre créée par son prédécesseur. Bloom dessine un parcours en six étapes qui retrace l'affranchissement du jeune poète de son "père " et son établissement à son tour comme poète fort. Il y a donc tout le long un enjeu de force et de pouvoir, et un risque concomitant d'écrasement.

La première étape de ce parcours est la plus intéressante ici : comment le jeune poète pourra-t-il commencer par s'affirmer face à l'œuvre qui menace de paralyser sa force créatrice? Par une lecture déviante qui opère un infléchissement minimal ${ }^{2}$. Bloom utilise les termes de misreading et misprision, et plus tard aussi misinterprétation pour nommer cet écart initial minimal qui marque cependant le pas décisif vers la propre œuvre du poète-fils. Tout commence donc, en tant qu'interprétation, par une mé-lecture, une mé-prise délibérée. Et chez Bloom ce " tout » est une question de pouvoir en tant que force créatrice. Le moment décisif se joue dans le préfixe mis- (mé-) qui, en principe négatif, prend une valeur positive comme une contestation (minimale) nécessaire pour ouvrir le champ où viendra s'inscrire une affirmation propre. Quelque lourdement œdipienne que soit cette théorie, elle articule, dès sa première phase et avec une lucidité remarquable, le moment de l'exercice d'un pouvoir dans l'acte déviant de

2. Bloom adopte de Lucrèce (De Rerum Natura) le terme de clinamen pour dire cet écart minimal mais décisif. 
l'interprétation. Il n'y va pas d'un geste révolutionnaire, mais d'un enjeu de survie dans le sens d'une continuation de l'activité créatrice.

À l'obsession œdipienne qui pèse sur cette théorie par ailleurs intéressante fait écho, dans le contexte nord-américain plus récent, une obsession plus générale du pouvoir. Elle colorie tout le débat sur la question de l'interprétation. C'est comme si le fait d'avoir reconnu l'enjeu de pouvoir dans tout acte d'interprétation obligeait les intellectuels à n'aborder l'herméneutique plus que sous le registre/signe du politique. Politics of interpretation est un des titres et un des syntagmes les plus utilisés dans les débats herméneutiques nord-américains ${ }^{3}$. Ceci s'inscrit, aux États-Unis dans le contexte assez pesant des politiques d'identité et de la rectitude politique ${ }^{4}$. C'est comme si l'incontournable "soupçon d'idéologie " des années'60 s'était mué, aux États-Unis du moins, en "soupçon de politique». Il s'agit alors partout de dépister le moment du pouvoir qui se manifeste dans l'acte d'interprétation. Au point où un observateur extérieur pourrait en conclure à une réduction de l'interprétation à son enjeu de pouvoir.

Dans le contexte d'une pensée post-coloniale qui, si articulée à partir d'un lieu périphérique, peut placer au banc des accusés les intellectuels nord-américains même les plus politiquement corrects, Édouard Glissant a poussé cette unidimensionnalité de l'interprétation à son extrême. En s'appuyant sur une logique étymologique quelque peu chancelante ${ }^{5}$, il démasque le power play herméneutique du " génie de l'Occident " : Et moi je dis que ce génie est un génie trompeur, parce que dans comprendre il y a l'intention de prendre, de sou-

3. Un exemple parmi beaucoup : le numéro spécial de Critical Inquiry $(9,1982)$ consacré à l'herméneutique sous le titre "Politics of Interpretation ».

4. Mais nous avons, au Québec, nos propres exemples de Politics of Interpretation, et ceci au sens tout à fait premier de cette expression : la manière de mêler la politique à l'interprétation d'un texte. Qu'on pense aux diverses interprétations partielles, partisanes, retorses, contresensuelles, etc auxquelles a été soumis le récent avis de la Cour Suprême du Canada sur la question de la sécession de la part de divers acteurs politiques, individuels et collectifs confondus.

5. «Dans (....) la notion de compréhension, « com-prendre», je prends avec moi, je comprends un être ou une notion, ou une culture, n'y a-t-il pas cette autre notion, celle de prendre, d'accaparer? » (p 126). 
mettre ce que l'on comprend, à l'aune, à l'échelle de sa propre mesure et de sa propre transparence »(p.126). Au concept de compréhension, il opposera alors l'appréciation des opacités de l'autre, et le "droit à l'opacité de tous les peuples »(p.128). Dans ce beau geste résonnent toutes sortes de rhétoriques, mais, pour l'herméneutique, il laisse ouverte une question importante : faut-il condamner à tout jamais l'herméneutique comme une machine de guerre de l'Occident colonisateur? Ou s'agit-il de développer une pratique herméneutique alternative, consciente de son enjeu de pouvoir intrinsèque et respectueux de l'altérité de qui il s'agit de comprendre?

Ma réponse est suggérée dans ce qui précède. Je m'oppose à ce qu'on réduise le vaste champ de l'herméneutique à une des dimensions qu'elle a privilégié dans certaines situations concrètes : la dimension du pouvoir. Et je plaiderai ici pour qu'on dissocie, sur le plan théorique, le type de pouvoir qui est exercé par l'interprète concret et le principe du travail interprétatif. Car si un potentiel de pouvoir se trouve constitutivement inscrit au niveau de ce principe, l'exercice concret de ce pouvoir dépend de la situation d'interprétation qui change de cas en cas. Finalement, il est important qu'on n'oublie pas toutes les autres dimensions du travail interprétatif : philologique, culturelle, sémiotique, éthique. Elles ne sont pas réductibles à du pouvoir, à moins d'une unidimensionnalité regrettable qui aurait pour effet l'appauvrissement de notre riche et complexe tradition herméneutique.

\section{Bibliographie}

Harold BLoom. The Anxiety of Influence. A Theory of Poetry. Oxford: Oxford University Press, 1975 ((C1973).

COLleCtif. Numéro spécial sur «The Politics of Interpretation», dans Critical Inquiry 9 (1982), pp 1-278.

Michel Foucault. L'ordre du discours. Paris : Gallimard, 1971.

Édouard GLISSANT. "Le chaos-monde, l'oral et l'écrit», dans Ralph LUDWIG (éd.), Écrire la parole de nuit. La nouvelle littérature antillaise. Paris : Gallimard, 1994, pp. 111-129.

Georges GuSDORF. Les origines de l'herméneutique. Paris : Payot, 1988. 
SAINT AUGUSTIN. Euvres de saint Augustin, vol. 11, $1^{\text {re }}$ série : Opuscules : XI. Le Magistère chrétien, Paris : Desclée de Brouwer et Cie, 1949.

Jean STAROBINSKI. "Le dîner de Turin », dans L'œil vivant II : La Relation critique. Paris : Gallimard, 1970, pp 88-169. 